\title{
Expression of cdc2 and p27(KIP1) phosphorylation in mitotic cells of the human retinoblastoma
}

\author{
SATORU KASE $^{1}$, KAZUHIKO YOSHIDA ${ }^{1}$, KAZUHIRO OHGAMI $^{1}$, KENJI SHIRATORI $^{1}$, \\ YUKARI SUZUKI $^{1}$, KEIICHI I. NAKAYAMA ${ }^{2}$ and SHIGEAKI OHNO ${ }^{1}$ \\ ${ }^{1}$ Department of Ophthalmology and Visual Sciences, Hokkaido University Graduate School of Medicine, N15, W7, \\ Kita-ku, Sapporo 060-8638; ${ }^{2}$ Department of Molecular and Cellular Biology, Medical Institute of Bioregulation, \\ Kyushu University, 3-1-1 Maidashi, Higashi-ku, Fukuoka 812-8582, Japan
}

Received September 26, 2005; Accepted November 18, 2005

\begin{abstract}
It was recently demonstrated that a lack of p27(KIP1) degradation resulted in the suppression of cdc 2 activity and consequent inhibition of entry into the M-phase. The aim of this study was to examine the distribution of phosphorylated p27(KIP1) on threonine 187 (T187-phospho-p27) and cdc2 in mitotic cells of human retinoblastoma, a malignant retinal neoplasm. Several T187-phospho-p27-immunopositive cells were observed in mitotic retinoblastoma cells, but not in the normal retina. Immunoreactivity for T187-phospho-p27 was located in the prophase and metaphase of mitotic tumor cells. In contrast, tumor cells in the anaphase showed no immunoreactivity for T187-phospho-p27. Nuclear expression of cdc2 was detected in many retinoblastoma cells, including mitotic cells. The immunoreactivity in mitotic cells was located in the prophase, as well as metaphase. In contrast, anaphase cells did not show immunoreactivity. Double staining demonstrated the same localization of T187-phospho-p27 and cdc2 in mitotic cells. These results suggest that p27(KIP1) interacts with cdc2 in the M-phase of human retinoblastoma cells.
\end{abstract}

\section{Introduction}

Retinoblastoma represents a malignant retinal neoplasm with neuroectodermal differentiation (1). Careful clinical and epidemiologic examination of hereditary and sporadic retinoblastomas gave rise to the so-called two-hit model that the development of cancer is linked to inactivation of both the maternal and paternal alleles of a particular tumor suppressor

Correspondence to: Dr Satoru Kase, Department of Ophthalmology and Visual Sciences, Hokkaido University Graduate School of Medicine, N15, W7, Kita-ku, Sapporo 060-8638, Japan

E-mail: kaseron@med.hokudai.ac.jp

Key words: cdc2, p27(KIP1), phosphorylation, mitosis, retinoblastoma gene (2). That the loss of $R b$ causes retinoblastoma in humans has been well established.

The cell cycle progression is controlled by a series of kinase complexes composed of cyclins and cyclin-dependent kinases (Cdks) (3). p27(KIP1) is a Cdk inhibitor (CKI) (4-7). It has been reported that $\mathrm{p} 27(\mathrm{KIP} 1)$ is specifically recognized by Skp2, and that this step was a rate-limiting component of the machinery that ubiquitinated and degraded p27(KIP1) $(8,9)$. Phosphorylation of p27(KIP1) on threonine (T) 187 by Cdk2 creates a binding site for a Skp2-containing E3 ubiquitinprotein ligase (10). In cycling cells, a fraction of p27(KIP1) is transiently present in the phosphorylated T187 form before degradation (11).

It was recently reported that $\mathrm{Rb}$ interacts with the $\mathrm{N}$ terminus of Skp2, interferes with Skp2-p27 interaction, and inhibits ubiquitination of p27(KIP1) (12). We have demonstrated that p27(KIP1) inhibited Cdk1 (historically known as cdc2) activity (13). Phosphorylation of p27(KIP1) at T187 (T187-phospho-p27) results in the ubiquitination and degradation of p27(KIP1) $(8,9,14)$, and a lack of degradation results in suppression of cdc 2 activity and consequent inhibition of entry into the M-phase (13). We recently demonstrated that T187-phospho-p27 immunoreactivity was noted in mitotic retinoblastoma cells (15).

In this study, we examined the distribution of T187phospho-p27 and cdc2 in human retinoblastoma.

\section{Materials and methods}

Operative specimens. Three cases, all Japanese girls, who were diagnosed with retinoblastoma clinically and underwent surgical resection at Hokkaido University Hospital, Sapporo, Japan, were examined. They had not received preoperative radio- and/or chemotherapy. Enucleation was performed in two cases, and orbital exenteration was done in the other case, which showed extrascleral invasion. Slides prepared from the retinoblastoma were washed in phosphate-buffered saline (PBS), fixed in ice-cold $4 \%$ paraformaldehyde in $0.1 \mathrm{M}$ borate buffer $(\mathrm{pH} 9.5)$ for $2 \mathrm{~h}$, and processed for paraffin sectioning. All studies conformed to the tenets of the Declaration of Helsinki. 


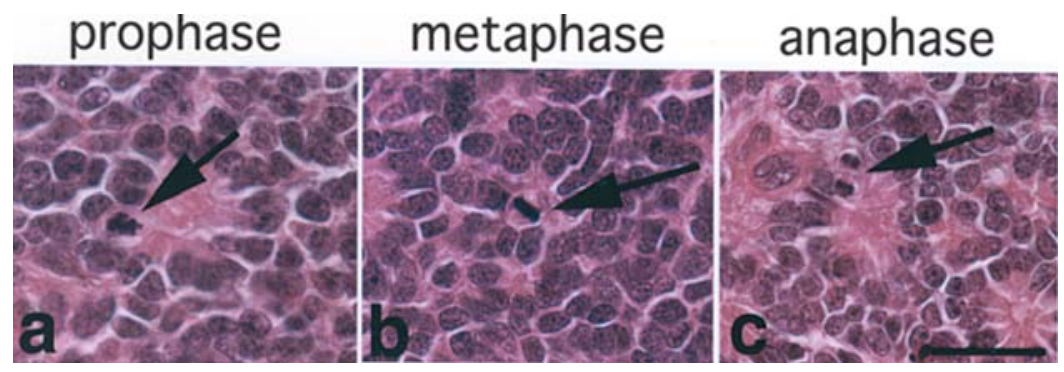

Figure 1. Mitotic figures in human retinoblastoma tissue (hematoxylin and eosin staining). In the prophase, the chromosomes condense to become visible microscopically (a, arrow). In the metaphase the chromosomes become maximally condensed and are lined up at the equator of the mitotic spindle (b, arrow). In the anaphase, sister chromatids located at the equator of the metaphase plate pull apart and begin their migration toward the opposite poles of the mitotic spindle (c, arrow). Bar indicates $50 \mu \mathrm{m}$.

\section{prophase metaphase anaphase}

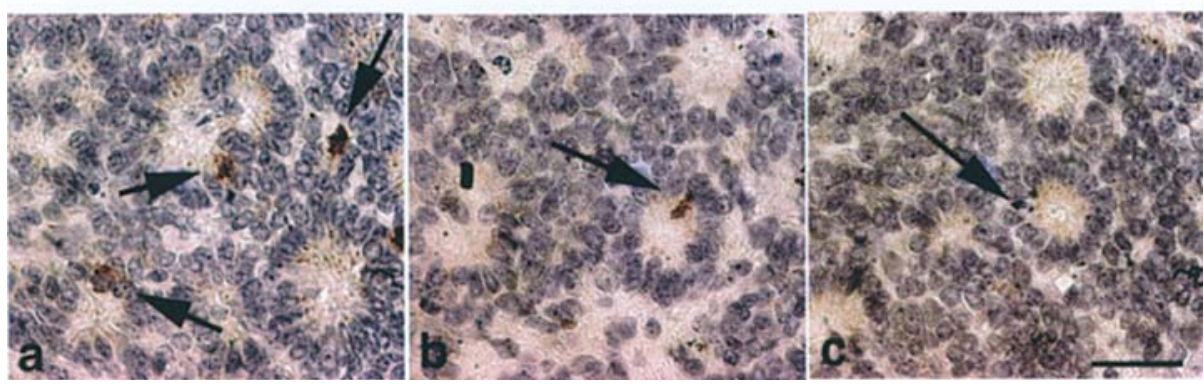

Figure 2. Immunodetection of threonine 187-phosphorylated p27(KIP1) (T187-phospho-p27) in each mitotic phase (prophase, metaphase and anaphase) of human retinoblastoma cells. Immunoreactivity for T187-phospho-p27 is located in the prophase (a, arrows) and metaphase (b, arrow) of mitotic tumor cells. Immunoreactivity for T187-phospho-p27 is less marked in anaphase tumor cells (c, arrow). Bar indicates $50 \mu \mathrm{m}$.
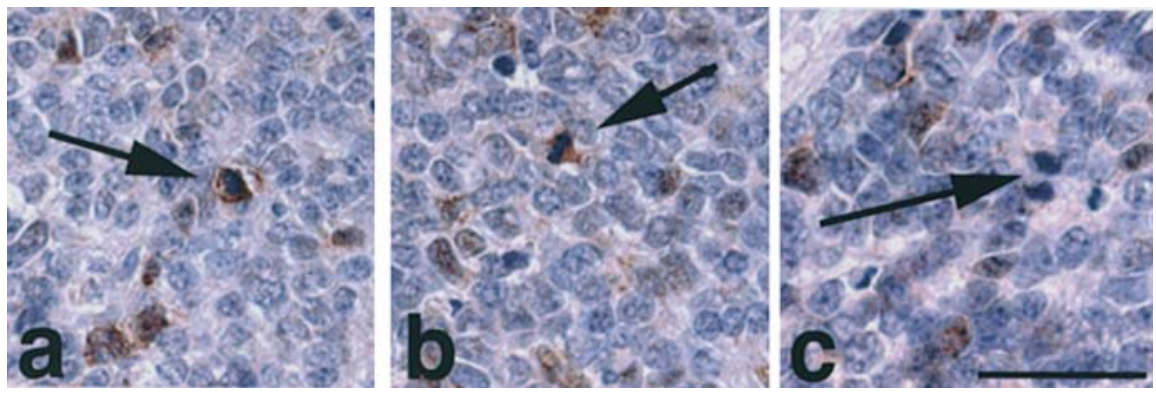

Figure 3. Expression of cdc2 in each mitotic phase (prophase, metaphase and anaphase) of human retinoblastoma cells. Expression of cdc2 in mitotic cells is apparent in the prophase (a, arrow), as well as the metaphase (b, arrow), retinoblastoma cells. In contrast, anaphase mitotic cells show no immunoreactivity (c, arrow). Bar indicates $50 \mu \mathrm{m}$.

T187-phospho-p27
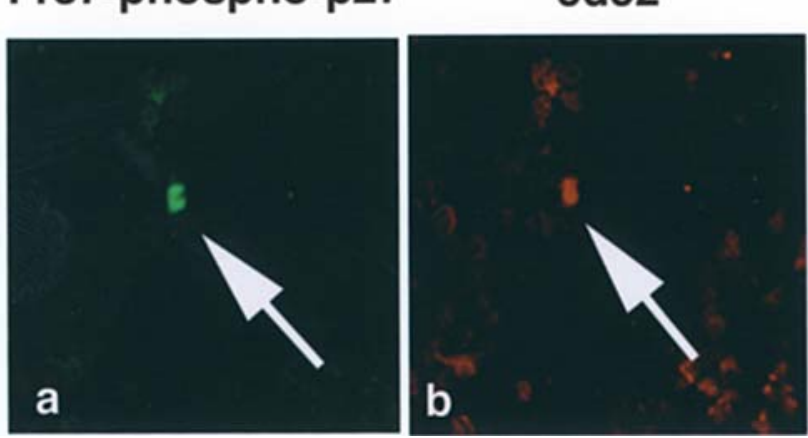

merge

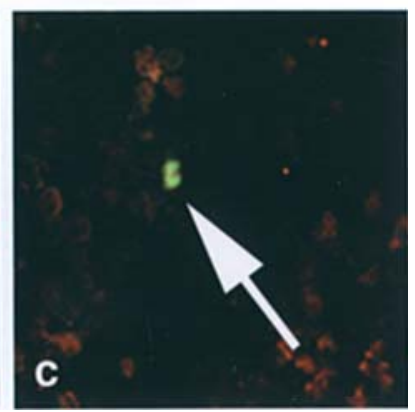

Figure 4. Immunoreactivity for threonine 187-phosphorylated p27(KIP1) (T187-phospho-p27) (a and c: green) and cdc2 (b and c: red) in the same retinoblastoma tissue. The cdc2-positive nuclei are distributed in the tumor cells, including mitotic cells (b), where T187-phospho-p27-immunopositive cells are also apparent (a-c, arrow). 
Immunohistochemistry. Dewaxed paraffin sections were immunostained using the streptavidin-biotin peroxidase complex method. Formalin-fixed, paraffin-embedded serial tissue sections were cut at $4 \mu \mathrm{m}$ thickness, and endogenous peroxidase activity was inhibited by immersing the slides in $0.3 \%$ hydrogen peroxide in methanol for $30 \mathrm{~min}$. As a pretreatment, microwave-based antigen retrieval was performed in $10 \mathrm{mM}$ citrate buffer ( $\mathrm{pH}$ 6.0). Then, non-specific binding of the primary antibody was blocked by incubating the slides in the blocking serum for $20 \mathrm{~min}$. The slides were serially incubated with anti-threonine 187 phosphorylated p27(KIP1) (T187-phospho-p27) polyclonal antibody (1:1000; Zymed, South San Francisco, CA), or anti-cdc2 monoclonal antibody (1:1000; Zymed) overnight at $4^{\circ} \mathrm{C}$, followed by the secondary antibody and biotin streptavidin complex for $30 \mathrm{~min}$ each, at room temperature. Immunoreactions were visualized with diaminobenzidine, and the sections were counterstained with hematoxylin. To examine the specificity of immunostaining, the primary antibody was replaced with mouse normal IgG or Tris-buffered saline. Control slides were invariably negative for immunostaining.

Double staining immunohistochemistry. The slides were dewaxed and rehydrated, and then rinsed in PBS twice for $10 \mathrm{~min}$ and incubated with the normal goat serum for $20 \mathrm{~min}$ and then with a mouse monoclonal antibody against cdc2 at room temperature for $2 \mathrm{~h}$. Binding of the primary antibody was localized with the FITC conjugated goat anti-mouse IgG (Jackson Immuno Research Laboratories Inc., West Grove, PA) for $30 \mathrm{~min}$. After three washes with PBS, tissue sections were then incubated with T187-phospho-p27 polyclonal antibody and biotin-conjugated anti-mouse $\operatorname{IgG}$ at room temperature for $60 \mathrm{~min}$. The section slides were examined using confocal laser microscope with double channel.

\section{Results}

In human retinoblastoma tissues, several mitotic figures were observed in tumor cells, which formed Flexner-Wintersteiner and Hormer-Right rosettes. Mitotic retinoblastoma cells without rosette formation were intermingled. Mitoses were not observed in the normal retina adjacent to retinoblastoma tissue. According to histological findings, mitotic retinoblastoma cells were classified into prophase, metaphase and anaphase (16). In mitotic cells, chromosomes were condensing and nucleolus disappeared, indicating the prophase (Fig. 1a). In the metaphase, the chromosomes became maximally condensed, and were lined up at the equator of the mitotic spindle (Fig. 1b). As shown in Fig. 1c, the anaphase begins when sister chromatids, located at the equator of the metaphase plate, pull apart and begin their migration toward the opposite poles of the mitotic spindle.

In order to determine the distribution of $\mathrm{p} 27(\mathrm{KIP} 1)$ phosphorylation on threonine 187 and cdc 2 in mitotic cells, immunohistochemistry was performed using human retinoblastoma specimens. Several T187-phospho-p27-immunopositive cells were observed in mitotic retinoblastoma cells, but not in the normal retina. At high magnification, immunoreactivity for T187-phospho-p27 was apparent in the prophase (Fig. 2a, arrow) and metaphase (Fig. 2b, arrow) of mitotic tumor cells. In contrast, tumor cells in the anaphase showed no immunoreactivity for T187-phospho-p27 (Fig. 2c, arrow).

Nuclear expression of cdc2 was detected in many retinoblastoma cells, including mitotic cells. At high magnification, the immunoreactivity in mitotic cells was located in the prophase, as well as the metaphase (Fig. 3a and b, arrow). In contrast, anaphase cells did not show immunoreactivity (Fig. 3c, arrow). Furthermore, no cdc2-positive nuclei were observed in the normal retina (data not shown).

Fig. 4 demonstrates the immunodetection of T187phospho-p27 (a and c: green), as well as cdc2 (b and c) in the retinoblastoma tissue using double staining immunohistochemistry. The cdc2-positive nuclei were distributed in the tumor cells, including mitotic cells (Fig. 4b), where T187phospho-p27-immunopositive cells were also noted (Fig. 4, arrows).

\section{Discussion}

Phosphorylation of $\mathrm{p} 27(\mathrm{KIP} 1)$ at $\mathrm{T} 187$ results in the ubiquitination and degradation of p27(KIP1) $(8,9,14)$, and a lack of the degradation leads to suppression of cdc2 activity and consequent inhibition of entry into the M-phase (13). We recently reported that immunoreactivity of p27(KIP1) was at an undetectable level, whereas that of T187-phospho-p27 was noted in mitotic cells of retinoblastoma $(15,17)$.

In this study, we examined the distribution of T187phospho-p27 and cdc2 in human retinoblastoma. As a first step, mitotic figures were classified into: the prophase, metaphase and anaphase according to morphological analysis (16) and the phosphorylation of p27(KIP1) by immunohistochemistry was analyzed. Nuclear immunoreactivity for T187phospho-p27 was apparent in the prophase and metaphase of mitotic tumor cells. In contrast, mitotic tumor cells in the anaphase showed no immunoreactivity for T187-phospho$\mathrm{p} 27$. These results suggest that p27(KIP1) is phosphorylated in the prophase and metaphase of the M-phase in retinoblastoma.

Nuclear immunoreactivity for cdc2 was detected in a variety of retinoblastoma cells, including mitotic cells. At high magnification, the expression was detected in mitotic cells, especially in the prophase, as well as metaphase mitotic retinoblastoma cells. In contrast, anaphase cells did not show immunoreactivity. These results indicated that cdc2 was expressed in the prophase and metaphase of the M-phase in retinoblastoma. Moreover, double staining immunohistochemistry demonstrated the same localization of cdc 2 and T187-phospho-p27 in mitotic retinoblastoma cells in this study. The presence of both T187-phospho-p27 and cdc2 in the prophase and metaphase of the M-phase suggests that p27(KIP1) interacts with cdc2 in the M-phase of human retinoblastoma cells, as we reported in mouse embryonic fibroblasts (13).

It was recently reported that $\mathrm{Rb}$ interacts with the $\mathrm{N}$ terminus of Skp2, interferes with Skp2-p27 interaction, and inhibits ubiquitination of p27(KIP1) (12). Inactivation of both copies of the $R b$ is associated with the development of retinoblastomas in humans (2). Regulation of cdc2 activity by p27(KIP1) should prove valuable in delineating the molecular pathways underlying mitosis in retinoblastoma. 


\section{Acknowledgements}

This study was supported by a grant for Research on Sensory and Communicative Disorders from The Ministry of Health, Labor, and Welfare, and by Grants-in-Aid for Scientific Research from The Ministry of Education, Culture, Sports, Science, and Technology (MEXT), Japan.

\section{References}

1. Albert DM: Historic review of retinoblastoma. Ophthalmology 94: 654-662, 1987

2. Knudson AG Jr: Mutation and cancer: statistical study of retinoblastoma. Proc Natl Acad Sci USA 68: 820-823, 1971.

3. Sherr CJ and Roberts JM: Inhibitors of mammalian G1 cyclindependent kinases. Genes Dev 9: 1149-1163, 1995.

4. Nourse J, Firpo E, Flanagan WM, Coats S, Polyak K, Lee MH, et al: Interleukin-2-mediated elimination of the p27Kip1 cyclindependent kinase inhibitor prevented by rapamycin. Nature 372: 570-573, 1994.

5. Reynisdottir I, Polyak K, Iavarone A and Massague J: Kip/Cip and Ink4 Cdk inhibitors cooperate to induce cell cycle arrest in response to TGF-beta. Genes Dev 9: 1831-1845, 1995.

6. Coats S, Flanagan WM, Nourse J and Roberts JM: Requirement of p27Kip1 for restriction point control of the fibroblast cell cycle. Science 272: 877-880, 1996.

7. Hirai A, Nakamura S, Noguchi Y, Yasuda T, Kitagawa M, Tatsuno I, et al: Geranylgeranylated rho small GTPase(s) are essential for the degradation of p27Kip 1 and facilitate the progression from $\mathrm{G} 1$ to $\mathrm{S}$ phase in growth-stimulated rat FRTL-5 cells. J Biol Chem 272: 13-16, 1997.
8. Carrano AC, Eytan E, Hershko A and Pagano M: SKP2 is required for ubiquitin-mediated degradation of the CDK inhibitor p27. Nat Cell Biol 1: 193-199, 1999.

9. Tsvetkov LM, Yeh KH, Lee SJ, Sun H and Zhang H: p27(Kip1) ubiquitination and degradation is regulated by the SCF(Skp2) complex through phosphorylated Thr187 in p27. Curr Biol 9: 661-664, 1999.

10. Skowyra D, Craig KL, Tyers M, Elledge SJ and Harper JW: F-box proteins are receptors that recruit phosphorylated substrates to the SCF ubiquitin-ligase complex. Cell 91: 209-219, 1997.

11. Vlach J, Hennecke S and Amati B: Phosphorylation-dependent degradation of the cyclin-dependent kinase inhibitor p27. EMBO J 16: 5334-5344, 1997.

12. Ji P, Jiang H, Rekhtman K, Bloom J, Ichetovkin M, Pagano M, et al: An Rb-Skp2-p27 pathway mediates acute cell cycle inhibition by $\mathrm{Rb}$ and is retained in a partial-penetrance $\mathrm{Rb}$ mutant. Mol Cell 16: 47-58, 2004.

13. Nakayama K, Nagahama H, Minamishima YA, Miyake S, Ishida N, Hatakeyama S, et al: Skp2-mediated degradation of p27 regulates progression into mitosis. Dev Cell 6: 661-672, 2004.

14. Sutterluty H, Chatelain E, Marti A, Wirbelauer C, Senften M, Muller U, et al: p45SKP2 promotes p27Kip1 degradation and induces $S$ phase in quiescent cells. Nat Cell Biol 1: 207-214, 1999.

15. Kase S, Yoshida K, Nakayama KI, Nakayama K, Ikeda H, Harada T, et al: Phosphorylation of p27(KIP1) in the developing retina and retinoblastoma. Int J Mol Med 16: 257-262, 2005.

16. Gartner L and Hiatt J: Color Textbook of Histology. W.B. Saunders Co., 1997.

17. Kase S, Yoshida K, Ohgami K, Shiratori K, Harada T and Ohno S: Expression of p27(KIP1) and cell proliferation in human retina and retinoblastoma. Anticancer Res 25: 3843-3846, 2005 . 